\title{
Changes in spinal cord excitability in a patient with rhythmic segmental myoclonus
}

\author{
V Di Lazzaro, D Restuccia, R Nardone, A Oliviero, P Profice, A Insola, P Tonali, \\ J C Rothwell
}

Istituto di Neurologia, Università Cattolica,

L go A Gemelli 8, 00168 Rome, Italy

V Di Lazzaro

D Restuccia

R Nardone

A Oliviero

P Profice

A Insola

P Tonali

J C Rothwell

Servizio di

Neurofisiopatologia,

CTO, Via S Nemesio

21, 00145 Rome, Italy

$A$ Insola

IRCCS “Casa Sollievo della Sofferenza" San Giovanni Rotondo,

Italy

P Tonali

MRC Human

Movement and

Balance Unit, The

National Hospital for

Neurology and

Neurosurgery, Queen

Square, London

WC1N 3BG, UK

J C Rothwell

Correspondence to:

Dr Vincenzo Di Lazzaro,

Istituto di Neurologia,

Università Cattolica, Lgo A

Gemelli 8, 00168 Rome,

Italy.

Received 8 March 1996

and in revised form

2 July 1996

Accepted 8 August 1996

\begin{abstract}
Paired stimulation of the common peroneal and posterior tibial nerve was used to study the recovery cycle of lumbosacral somatosensory evoked potentials in 10 control subjects and in one patient with rhythmic segmental myoclonus of the leg involving the L2-L4 myotomes. In normal subjects the peripheral nerve volley in the cauda equina had recovered at an interstimulus interval of $3 \mathrm{~ms}$ whereas the postsynaptic dorsal horn potential was reduced to about $60 \%$ of its control size. Similar results were found in the patient after posterior tibial nerve but not common peroneal nerve stimulation. The second, which evokes afferent input to the affected lumbar segments, produced facilitation of the postsynaptic response at $3 \mathrm{~ms}$. This finding suggests that the physiological suppression of dorsal horn interneurons which usually takes place after paired stimulation fails to occur in segmental myoclonus. This may indicate that dorsal horn interneurons are abnormally hyperactive and are involved in the pathophysiology of spinal myoclonus.
\end{abstract}

(F Neurol Neurosurg Psychiatry 1996;61:641-644)

Keywords: myoclonus; spinal cord; evoked potentials

Myoclonus may be the result of abnormal activity in many different parts of the CNS. That of spinal origin is distinguished by the fact that $(a)$ the jerking is usually rhythmic, $(b)$ the muscles involved are innervated by a restricted segment of the cord, and $(c)$ there is no sign that the jerks are preceded by EEG activity in the contralateral sensorimotor cortex.

The pathophysiology of spinal myoclonus remains speculative. Histological studies have shown a striking reduction in the number of small and medium sized neurons in the posterior horns of the lumbar cord with relative sparing of large neurons in the anterior horns. ${ }^{1}$ Taken together with EMG studies that have demonstrated the absence of denervation potentials, ${ }^{23}$ this suggests that relatively normal $\alpha$ motor neurons are driven to discharge by abnormal activity in spinal circuits which lack input from the missing interneurons.

To test this hypothesis we evaluated dorsal horn function in one patient with spinal myoclonus by recording the spinal evoked potentials produced by stimulation of the common peroneal and posterior tibial nerves. Spinal responses in humans are similar to those described in monkeys, and are thought to reflect the postsynaptic neuronal response to inputs conveyed by group I and II peripheral afferent fibres in Rexed layers IV and V. ${ }^{4}$ These responses are generated by static transverse dipolar sources in the grey matter of the spinal cord. ${ }^{5}$ We recorded the response to single stimuli and also to pairs of stimuli to study the recovery cycle of excitability changes within the dorsal horn.

\section{Patient}

A previously healthy woman aged 39 presented with a two month history of lower limb jerking. She had bilateral, rhythmic, involuntary jerks of proximal lower limb muscles. These jerks were intensified by emotional stress and ceased during sleep. Strength, tone, and tendon reflexes were normal and plantar responses were bilaterally flexor. Neurological examination was otherwise normal. Full blood examination was normal. Magnetic resonance imaging of the CNS was normal, as were motor and sensory conduction studies. Needle EMG recording from leg muscles disclosed repetitive discharges of motor units at a rate of about $0.4 \mathrm{~Hz}$ in the iliopsoas, adductor, and rectus femoris muscles on either side. No signs of denervation were found. The EEG, the blink reflex, and the recovery cycle of the blink reflex were normal. Motor evoked potentials after cortical and paravertebral magnetic stimulation were of normal latency. Standard somatosensory evoked potentials (SEPs) after stimulation of the median, common peroneal, and tibial nerve were of normal amplitude and latency.

\section{Methods}

For SEP recording, the patient lay on a couch in a warm and semidarkened room. Stimuli $(0.2 \mathrm{~ms}$ duration, $2 \mathrm{~Hz})$ were delivered through skin electrodes at the popliteal fossa 


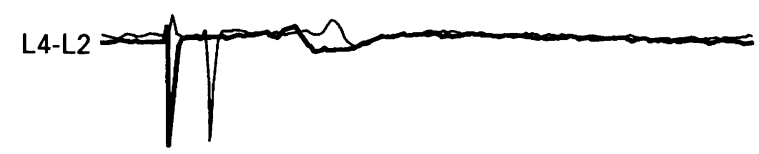

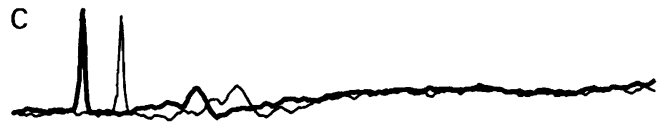

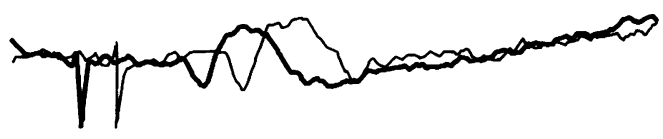

Posterior tibial nerve (ankle)

B
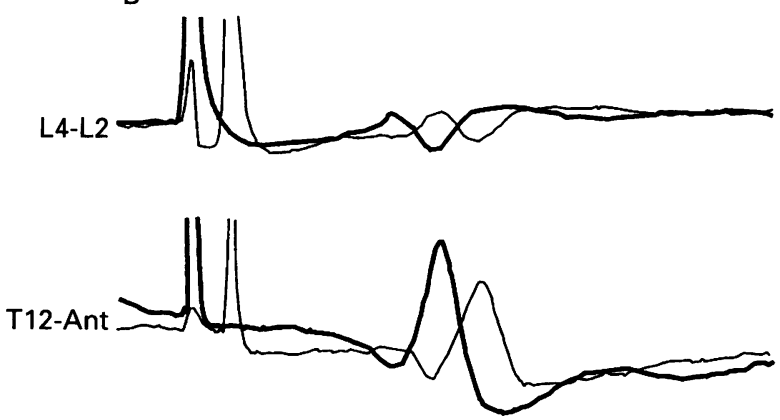

D
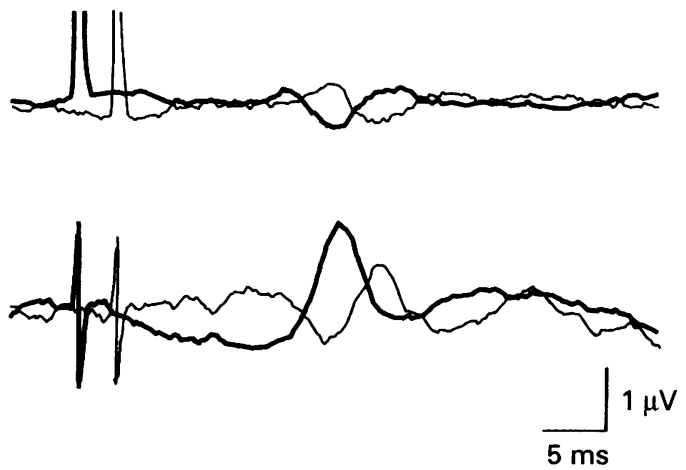

Figure 1 Paired stimulation in a control subject and in the patient. (A). Paired stimulation of common peroneal nerve with an interstimulus interval of $3 \mathrm{~ms}$ in a normal subject. The conditioned cauda equina response is of comparable amplitude to the control response (thicker line). The conditioned N14 is about $60 \%$ of the responses obtained with a single stimulus (thicker line). (B) Paired stimulation of posterior tibial nerve with an interstimulus interval of $3 \mathrm{~ms}$ in a normal subject. The conditioned cauda equina response is of comparable amplitude to the control response (thicker line). The conditioned N24 is about $60 \%$ of the responses obtained with a single stimulus (thicker line). (C) Paired stimulation of common peroneal nerve with an interstimulus interval of $3 \mathrm{~ms}$ in the patient. The conditioned cauda equina response is of comparable amplitude to the control response (thicker line). The conditioned N14 is about $120 \%$ of the responses obtained with a single stimulus (thicker line). (D) Paired stimulation of posterior tibial nerve with an interstimulus interval of $3 \mathrm{~ms}$ in the patient. The conditioned cauda equina response is of comparable amplitude to the control response (thicker line). The conditioned N24 is about $70 \%$ of the responses obtained with a single stimulus (thicker line).

for the common peroneal nerve and at the ankle for the posterior tibial nerve; stimulus intensity was adjusted to be slightly above motor threshold. The filter bandpass was 30-3000 Hz. Responses were averaged with an analysis time of $50 \mathrm{~ms}$. Samples with excessive interference were automatically edited out of the average. Two averages of 2048 trials each were obtained. The recording electrodes (impedance below $5 \mathrm{kohm}$ ) were placed over the spinal processes of L4 and T12. The L4 electrode was referred to L2 to record the response generated by the ascending volley of impulses in the cauda equina.$^{67}$ For recording the spinal potential, which we labelled as N14 for the common peroneal nerve and as N24 for the posterior tibial nerve, we connected grid 1 of the amplifier to the T12 electrode and grid 2 to an electrode placed over the anterior abdomen. The rationale for this montage has been discussed in detail in a previous study ${ }^{8}$ Briefly, it permits the selective recording of the activity generated by the transverse dipolar source located in the lumbosacral spinal cord ${ }^{9}$; moreover, it can cancel noise from the ECG activity that is picked up by both T12 and anterior electrodes. The amplitudes of responses were measured peak to peak.

In a preliminary study on three normal subjects we determined the recovery curves of cauda equina and spinal responses by delivering pairs of stimuli of equal intensity to the common peroneal nerve or posterior tibial nerve with interstimulus intervals of $2,3,4$, and 5 ms. As peripheral nerve excitability, judged by the cauda equina potential, had recovered by 3 $\mathrm{ms}$ (fig 1), the patient and $10 \mathrm{control}$ subjects (mean age 38.1 (SD 4.7) years) were studied with this interstimulus interval. To measure the recovery of the conditioned response, the test response recorded using a single stimulus was subtracted off line from the responses recorded using paired stimuli. The test in controls and in the patient was performed after stimulation of nerves of the right side. The upper limit of the conditioned response was defined as the mean $+2.5 \mathrm{SDs}$ of the normal values.

\section{Results}

TIME COURSE OF SEP RECOVERY IN THREE NORMAL SUBJECTS

The recovery cycle of the cauda equina and spinal responses in three normal subjects were 
Figure 2 (A) Recovery cycle of the cauda equina response ( ) and N14 response (-) to paired stimulation of common peroneal nerve. Each trace is the mean of responses recorded in three different subjects; $S D$ are indicated. The conditioned response amplitude is expressed as a percentage of response obtained with a single stimulus. Cauda equina refractory period ends at 3 ms interstimulus interval with a full recovery of the response. At this interval there is still a pronounced depression of conditioned N14. (B) Recovery cycle of the cauda equina response ( $\bullet$ ) and $N 24$ response (O) to paired stimulation of the posterior tibial nerve. Each trace is the mean of responses recorded in three different subjects; $S D$ are indicated. The conditioned response amplitude is expressed as a percentage of the response obtained using a single stimulus. Cauda equina refractory period ends at 3 ms interstimulus interval with a full recovery of the response. At this interval there is still a pronounced depression of conditioned N24.

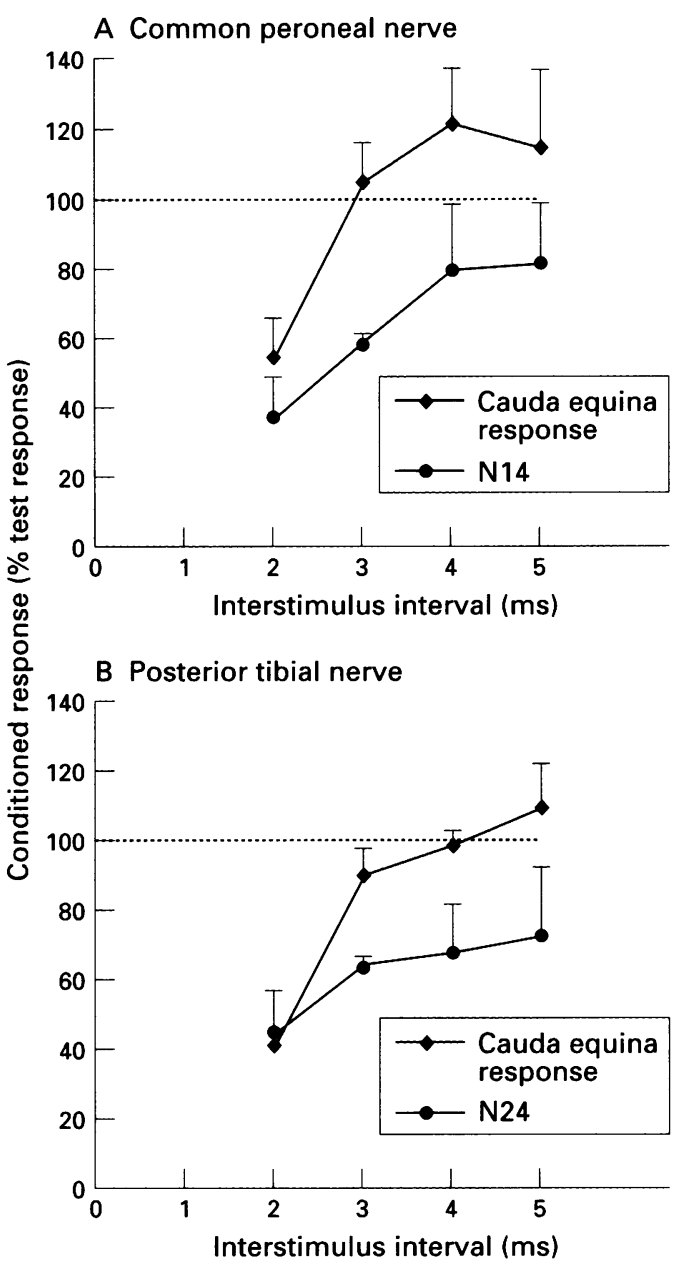

constructed from pairs of equal stimuli given to the common peroneal nerve or posterior tibial nerve with an interstimulus interval ranging from 2 to $5 \mathrm{~ms}$. Figure 1 shows typical examples of normal responses to both a single stimulus, and to the second stimulus of a pair with an interstimulus interval of $3 \mathrm{~ms}$. The response to the second stimulus of a pair has been constructed by subtracting the response to a single shock from the total response to a pair of shocks. Figure 2 shows the mean results from the three normal subjects. Cauda equina potential recovered completely at $3 \mathrm{~ms}$. At this interstimulus interval spinal responses evoked by the second stimulus of the pair were still suppressed. Spinal responses fully recovered at an interstimulus interval of $5 \mathrm{~s}$.

COMPARISON OF SEP RECOVERY AT 3 MS IN THE PATIENT VERSUS 10 NORMAL SUBJECTS

In control subjects, using an interstimulus interval of $3 \mathrm{~ms}$ the mean amplitude of the cauda equina response was $95.5 \%(19 \%)$ of the response evoked with a single stimuli for the posterior tibial nerve and $97 \%(14 \%)$ for the common peroneal nerve. The mean amplitude of N24 after stimulation of the posterior tibial nerve was $61 \%(4.6 \%)$ of the response evoked with a single stimuli, and the N14 after common peroneal nerve stimulation was $60.6 \%(5 \cdot 9 \%)$. Thus the upper limit for a conditioned spinal response was $72.9 \%$ for the posterior tibial nerve and $75.4 \%$ for the common peroneal nerve.
In the patient we found a normal inhibition of conditioned response after posterior tibial nerve stimulation with a conditioned response of $69.6 \%$ of the test response and a cauda equina response of $98 \%$ of the test (fig 1). Paired stimuli to the common peroneal nerve produced no inhibition of the conditioned response and on the contrary resulted in an increase of the amplitude of the $\mathrm{N} 14$ that was $121 \%$ of the test response (fig 1 ). The cauda equina response was $100 \%$ of the test. The amplitude and rhythm of the jerks were not affected by the nerve stimulation.

\section{Discussion}

Our patient had typical rhythmic segmental myoclonus. ${ }^{10}$ There was no evidence of cortical or brainstem hyperexcitability; cortical SEPs were of normal amplitude and the recovery cycle of the blink reflex was normal. The segmental distribution of jerks and the absence of any excitability changes in cerebral cortex or brainstem strongly suggest that the jerks had a spinal origin.

The pathophysiology of spinal myoclonus remains speculative. As outlined in the introduction, several authors have suggested that the underlying pathophysiology involves spinal interneurons rather than $\alpha$ motor neurons and this has been supported by histological evidence in one case. ${ }^{1}$ To date, however, there has been little evidence of a physiological abnormality which might parallel the anatomical changes. Davis et al ${ }^{1}$ showed in one patient that stimulation of the common peroneal nerve on one side could evoke abnormal short latency responses in the opposite leg. It is likely that such responses are caused by hyperexcitability of interneuronal connections between the two sides of the cord which under normal circumstances are relatively suppressed. The present data provide further evidence for physiological hyperexcitability in the spinal cord.

In normal subjects, the spinal potential of the dorsal horn produced by the second stimulus of a pair to either the common peroneal nerve or the posterior tibial nerve was suppressed to $60 \%$ of its control value when the interval between the shocks was $3 \mathrm{~ms}$. At the same interstimulus interval, cauda equina responses had completely recovered to the control level, suggesting that spinal rather than peripheral mechanisms were responsible for the inhibition. This finding is in agreement with previous studies on the recovery cycle of spinal SEPs. ${ }^{11}$ Our patient had segmental myoclonus involving iliopsoas, quadriceps, adductor muscles, and thus the L2-L4 myotomes. ${ }^{12}$ Spinal responses to a single nerve stimulus were clear from both the posterior tibial nerve and the common peroneal nerve. The main changes occurred with paired stimuli. After common peroneal nerve stimulation, the response to the second stimulus of the pair was enhanced, whereas it was suppressed to normal after posterior tibial nerve stimulation. The dorsal root innervation of the common peroneal nerve is from lumbar myelomeres ${ }^{13}$ 
from the same spinal segments as those involved in the genesis of the myoclonus whereas the sacral myelomeres mainly contribute to the posterior tibial nerve effect. ${ }^{1415}$ We conclude that there was local hyperexcitability of the mechanisms responsible for the dorsal horn potential after common peroneal nerve stimulation.

The enhancement of the conditioned common peroneal nerve spinal response in our patient suggests that in segmental spinal myoclonus dorsal horn interneurons are abnormally hyperactive. This finding, in association with an EMG study in our patient and in previous studies ${ }^{23}$ that showed no denervation in involved muscles, strongly suggests that in spinal myoclonus the underlying pathophysiology involves dorsal horn interneurons.

From a clinical point of view, the data in our patient suggest that study of spinal recovery curves is capable of confirming the spinal origin of excitability changes in patients with segmental myoclonus just as the recovery cycle of cortical SEPs can for forms of myoclonus originating in the cerebral cortex.

1 Davis SM, Murray NMF, Diengdoh JV, Galea-Debono A, Kocen RS. Stimulus sensitive spinal myoclonus. F Neurol Neurosurg Psychiatry 1981;44:884-8.

2 Hopkins AP, Michael F. Spinal myoclonus. I Neurol Neurosurg Psychiatry 1974;37:1112-5.

3 Nohl M, Doose H, Gross-Selbeck G, Jensen HP. Spinal myoclonus. Eur Neurol 1978;17:129-35.

4 Beall JE, Applebaum AE, Foreman RD, Willis WD. Spinal cord potentials evoked by cutaneous afferents in the monkey. F Neurophysiol 1977;40:199-211.

5 Jeanmonod $D$, Sindou $M$, Mauguière $F$. Three transverse dipolar generators in the human cervical and lumbosacral dorsal horn: evidence from direct intraoperative recordings on the spinal cord surface. Electronencephalogr recordings on the spinal cord surface

6 Magladery JW, Porter WE, Park AM, Teasdall RD. Electrophysiological studies of nerve and reflex activity in normal man, IV. The two-neurone reflex and identification of certain action potentials from spinal roots and cord. Bulletin of fohns Hopkins Hospital 1951;88:499-519.

7 Cracco JB, Cracco RQ, Graziani LJ. The spinal evoked response in infants and children. Neurology 1975;25: 31-6.

8 Restuccia D, Di Lazzaro V, Valeriani M, Colosimo C, Tonali P. N24 spinal response to tibial nerve stimulation and magnetic resonance imaging in lesions of the lumbosacral spinal cord. Neurology 1993;43:2269-75.

9 Desmedt JE, Cheron G. Spinal and far-field components of human somatosensory evoked potentials to posterior tibial nerve stimulation analysed with oesophageal derivations nerve stimulation analysed with oesophageal derivations
and non-cephalic reference recording. Electronencephalogr Clin Neurophysiol 1983;56:635-51.

10 Bressman S, Fahn S. Essential myoclonus. In: Fahn S, Marsden CD, Van Woert MH, eds. Myoclonus. Advances in neurology. Vol 43. New York: Raven Press, 1986: 287-94.

11 Saito T, Yamada T, Hasegawa A, et al. Recovery functions of common peroneal, posterior tibial and sural nerve somatosensory evoked potentials. Electroencephalogr Clin Neurophysiol 1992;85:337-44.

12 Wilbourn AJ. The value and limitations of electromyographic examination in the diagnosis of lumbosacral radiculopathy. In: Hardy RW, ed. Lumbar disc disease. New York: Raven Press, 1982:65-109.

13 LH Bannister, MM Berry, P Collins, et al. Nervous system. In: PL Williams, LH Bannister, MM Berry, P Collins, M In: PL Williams, LH Bannister, MM Berry, P Collins, $M$ Dyson, JE Dussek, MWJ Ferguson, eds. Gray's anatomy.

14 Foester, O. Spezielle Anatomie und Physiologie del peripheren Nerven. In: Lewandowsky M, ed. Handubuch del Neurologie, Erganzungsband, zweiter Teil. Berlin: Springer, 1929:785-974

15 Chiappa KH. Short-latency somatosensory evoked potentials: methodology. In: Chiappa $\mathrm{KH}$, ed. Evoked potentials in clinical medicine. New York: Raven Press, 1985: 203-312. 